\author{
Commentary on: Jansen F., Lumb D., Altieri B., et Al., 2001, A\&A, 365, L1; \\ Strüder L., Briel U., Dennerl K., ET Al., 2001, A\&A, 365, L18; \\ Turner M. J. L., Abbey A., Arnaud M., et Al., 2001, A\&A, 365, L27
}

\title{
A decade of X-ray astronomy with XMM-Newton
}

\author{
M. Güdel
}

Institute of Astronomy, ETH Zurich, 8093 Zurich, Switzerland e-mail: guedel@astro.phys.ethz.ch

The XMM-Newton X-ray observatory (previously known as the X-Ray Multi-Mirror Mission, XMM) was launched on 10 December 1999 by the first commercial Ariane 5 launch into a highly eccentric, $48 \mathrm{~h}$ orbit. XMM-Newton has been a key project of the European Space Agency (ESA), defined as a cornerstone mission in ESA's Horizon 2000 program. Nearly ten years after its launch, XMM-Newton operates flawlessly and has become a workhorse at the forefront of X-ray astronomy. The paper by Jansen et al. (2001), written by some of the key people in the project, is the defining post-launch paper for the XMM-Newton observatory, summarizing the key features of the satellite, various parts of the ground segment, software, and calibration. It is the opening article in a series of papers in the same A\&A special issue (Vol. 365) that present more details on the instruments, software, and calibration, together with an impressive suite of "first results" papers from across all fields of X-ray astronomy. The papers by Strüder et al. (2001) and Turner et al. (2001) describe the X-ray imaging cameras onboard (XMM-Newton) in detail.

The concept of XMM-Newton and its scientific goals were developed and discussed in the early eighties with great foresight. Routine X-ray astronomy in the $\approx 0.1-10 \mathrm{keV}$ range had been established only a few years before, and a suite of smaller - and highly successful - X-ray satellites were still being developed at the time, for launch later in the decade and in the nineties.

Among XMM-Newton's main strengths is the simultaneous operation of six science instruments, providing high X-ray throughput, important redundancy, and unprecedented spectroscopic capabilities. Three independent Wolter I telescopes consisting of 58 nested mirror shells each offer an unparalleled effective area of $\approx 2000 \mathrm{~cm}^{2}$ (at $\approx 1 \mathrm{keV}$ ) in conjunction with the three European Photon Imaging Cameras (EPIC). The EPIC cameras provide a wide (half-degree) field of view, with a point-spread function diameter of about $5^{\prime \prime}$ (FWHM) and lowresolution spectroscopy.

The advent of high-resolution X-ray spectroscopy with XMM-Newton and NASA's Chandra X-ray Observatory (CXO) has marked a breakthrough in X-ray astronomy. XMM-Newton's two reflection grating spectrometers (RGS) offer by far the highest effective area so far available for X-ray grating spectroscopy below $\approx 1 \mathrm{keV}$, giving access to pivotal line diagnostics for many astrophysical sources.

Finally, XMM-Newton operates a very sensitive optical monitor (OM) that can be used in conjunction with various optical and ultraviolet filters for imaging, but also in spectroscopy (grism) mode. The simultaneous operation of the X-ray imaging and spectroscopy instruments plus an optical instrument has been one of the significant assets of the XMM-Newton mission.

The scientific achievements of XMM-Newton are much too numerous to summarize within the available space. The Jansen et al. introductory paper and the Strüder et al. and Turner et al. EPIC papers have been followed by an impressive number of scientific publications, averaging more than 200 refereed publications per year based partially or completely on XMM-Newton studies, with a cumulative number of 2060 papers by the end of March 2009. Approximately 1500-2000 scientists define the user community of XMM-Newton world-wide, obtaining data in the framework of annual calls for observing time proposals and subsequent peer-review processes. A biased sample of selected highlights from various research fields must suffice to illustrate XMM-Newton's scientific contributions to X-ray astronomy; for references to these and many further contributions, see, e.g., the proceedings edited by Wilson (2006) and by Schartel (2008).

Among XMM-Newton's targets in our Solar System, Jupiter showed prominent auroral soft emission due to charge exchange, but also revealed a new, variable hard component consistent with bremsstrahlung from electrons precipitating from the magnetosphere. Similarly, two X-ray components were discovered from Mars: one due to upper-atmospheric fluorescent scattering of solar X-rays and the other due to exospheric solar wind charge exchange.

Research on cool stars has profited from high-resolution spectroscopy. Spectroscopically determined physical parameters, such as plasma density or opacity due to resonant scattering, have contributed to models of stellar coronal structure. The first-light observation of the RGS discovered a now muchdiscussed abundance anomaly in stellar coronae, the so-called inverse first-ionization potential effect, pointing to an element fractionation process in the lower atmospheric layers at variance with observations of the solar corona. The long operational lifetime of XMM-Newton has also uncovered activity cycles in a few solar-like stars, potentially helping theoretical modeling of magnetic dynamos.

XMM-Newton has been very effective in surveying starforming regions across wide fields. Among various other findings, RGS studies led to the discovery of a "soft excess" in accreting T Tauri stars, related to cool plasma probably associated with the accretion streams. EPIC spectroscopic studies revealed variable fluorescent $\mathrm{Fe}$ emission probably originating in the circumstellar disk of a protostar, while EPIC imaging identified 
a hot plasma bubble pervading the Orion Nebula star-forming region.

Hot stars develop prominent winds in which shocks heat gas to $>1 \mathrm{MK}$. Quantitative analysis of high-resolution spectra with resolved line profiles have led to new insights into the wind structure (porous winds, etc.) and new debates on the location of the X-ray sources within the winds. Colliding winds between massive stars, but also magnetically confined winds around single stars, have contributed to a deeper understanding of stellar mass loss and environments of high-mass stars.

Supernovae (SN) in other galaxies have been the subject of "target of opportunity" studies. They show up as particularly bright sources in the ultraviolet bands covered by the OM, although XMM-Newton was successful in detecting X-rays as well. The X-rays have been used to characterize both forward and reverse shocks in the expanding shells. A new class of type $1 \mathrm{SN}$ with massive and rather young (100 Myr) progenitors was discovered in the course of these studies.

Similarly, gamma-ray bursts (GRB) have successfully been caught in action. Observations not only monitored the bursts themselves, but also discovered a dust-scattered X-ray halo around a GRB, permitting a precise measurement of the distance to the scattering dust within our galaxy.

In supernova remnants (SNRs) spatially resolved X-ray emission has been used to map the distribution of shocked synthesized elements in SN ejecta. Similar studies of planetary nebulae $(\mathrm{PN})$ have given new evidence on the origin and heating of the material. Galactic SNRs observed with the EPIC cameras further reveal clear evidence of non-thermal synchrotron radiation in the outermost regions where magnetic fields must be amplified and particles are accelerated to $\mathrm{TeV}$ energies.

XMM-Newton has offered interesting new clues about neutron stars (NS). A synchrotron bow shock was imaged in the vicinity of the Geminga pulsar, allowing a probe of the interstellar medium through which the NS travels, but also the injection energy of accelerated electrons and the shock magnetic fields. Spectroscopy of the NS themselves has shown various thermal and power-law components partly originating in X-ray hot spots whose dimensions can be determined, and also uncovered resonant cyclotron absorption permitting estimates of magnetic field strengths. If pulsations are detected in X-rays, they contribute to determining spin-down/energy loss physics in various systems, including magnetars in which the decay of the magnetic field is the ultimate energy source. XMM-Newton observations moreover led to the first discovery of a relativistic Fe line from the inner accretion disk in a low-mass X-ray binary. Observations of gravitational redshift related to an NS in a low-mass X-ray binary were used to derive the equation of state for NS.

Studies of galactic black holes (BHs) have now discovered an example in a globular cluster. Spectral studies of broad and skewed $\mathrm{Fe} \mathrm{K} \alpha$ lines have indicated the presence of rotating (Kerr) BHs and suggested that rotational energy can be extracted from BHs. The mass of the Galactic center $\mathrm{BH}$ has been narrowly confined by making use of quasi-periodic oscillations (QPOs). Such QPOs have for the first time also been found in active galactic nuclei (AGN), providing important links to a unified theory of accretion onto BHs.

Surveys of nearby galaxies with their diverse X-ray populations, including XRBs, SNRs, and novae, have profited greatly from the large field of view of XMM-Newton. A particular survey was performed for the Andromeda galaxy, providing an example with which to interpret more distant, normal galaxies and their evolution at various redshifts as observed in wide-field surveys.
Relativistically broadened emission lines have been one focus of research in the field of AGN; these lines offer crucial diagnostics for the inner part of the accretion disks and the spin of the central black holes. A special example is the detection of modulation of transient $\mathrm{Fe} \mathrm{K} \alpha$ features in AGN.

Observations of photoionized warm and hot absorbers related to AGN outflows may diagnose the AGN environment down to the immediate BH neighborhood. Strongly linedominated, high-resolution spectra of Seyfert-2 galaxies have provided further support for photoionized material, perhaps related to these absorbers. The softest part of AGN X-ray spectra often reveals a soft X-ray excess for which new interpretations have been proposed, such as relativistically smeared wind absorption or reflection from an ionized disk.

Spectral and imaging studies of galaxy cluster gas have helped to characterize fundamental physical parameters of clusters (e.g., mass, density, entropy) and also to determine its composition and the origin of the heavy elements, e.g., from different types of SNs. High-resolution spectroscopy has revealed a deficiency of cool gas in the central part of the clusters, suggesting inhibited cooling and leading to significant modifications of the standard "cooling flow" models.

Measurements related to the temperature-luminosity relation of galaxy clusters have provided important input to cosmological issues, such as the evolution of cosmological parameters. Deep and wide-field surveys, such as the XMM-COSMOS 2square degree survey, have contributed to the characterization of the X-ray background and the population of obscured starburst galaxies and AGN. XMM-Newton has also been used to search for the warm-hot intergalactic medium (WHIM), with the aim of providing important new constraints on the matter/dark matter budget in the local Universe.

While XMM-Newton's capabilities overlap those of CXO, complementary strengths of the two missions (in terms of angular resolution, effective area, time resolution, etc.) have made joint observations worthwhile, resulting in considerable amounts of added science. The same is true for numerous joint observations with other, ground-based optical, near-infrared, and radio observatories.

XMM-Newton's highly successful mission has relied not only on frontier instrumentation but also on an efficient ground segment, consisting of the Mission Operations Centre (MOC, Darmstadt, Germany), the Science Operations Centre (SOC, Villafranca, Spain), and the Science Survey Centre (SSC, Leicester, UK) responsible for, respectively, spacecraft operations, processing of science data and distribution to the community, and generation of standard science products, among other things. An additional guest observer facility has been supporting observers in the United States.

Current assessment of the (excellent) instrument health and onboard consumables will support full operation of XMMNewton until 2018, then completing nearly two decades of intense observations of the X-ray sky.

Acknowledgements. I warmly thank the XMM-Newton Project Scientist, Norbert Schartel (SOC), for useful information. XMM-Newton is an ESA science mission with instruments and contributions directly funded by ESA Member States and NASA.

\section{References}

Jansen, F., Lumb, D., Altieri, B., et al. 2001, A\&A, 365, L1 Schartel, N., \& Ehle, M. (eds.) 2008, Astron. Nachrichten, 329, 111 Strüder, L., Briel, U., Dennerl, K., et al. 2001, A\&A, 365, L18 Turner, M. J. L., Abbey, A., Arnaud, M., et al. 2001, A\&A, 365, L27 Wilson, A. (ed.) 2006, The X-ray Universe 2005, ESA SP-604, 999 